\title{
BOREL STRUCTURES AND BOREL THEORIES
}

\author{
GREG HJORTH AND ANDRÉ NIES
}

\begin{abstract}
We show that there is a complete, consistent Borel theory which has no "Borel model" in the following strong sense: There is no structure satisfying the theory for which the elements of the structure are equivalence classes under some Borel equivalence relation and the interpretations of the relations and function symbols are Borel in the natural sense.

We also investigate Borel isomorphisms between Borel structures.
\end{abstract}

\section{INTRODUCTION}

The completeness theorem states that each consistent first-order theory $T$ has a model $\mathcal{M}$ no larger than the size of the language. If the language is countable then $\mathcal{M}$ can be defined from $T$. If the language is effectively given then the elementary diagram of $\mathcal{M}$ is computable relative to $T$. On the other hand, if the language is uncountable, the proof of the completeness theorem relies on the axiom of choice.

We investigate theories and structures the size of the continuum. Here a natural way to impose an effectivity condition is to use a first order language that can be seen as a standard Borel space, and to require that the theory is Borel. A Borel structure is one where the elements of the structure are equivalence classes under some Borel equivalence relation $E$ and the interpretations of the relations and function symbols are Borel. For example, the field of reals with constants naming each element, and a unary function symbol naming each continuous function, is a Borel structure. A further example of a Borel structure is the Boolean algebra $\mathcal{P}(\omega)$ modulo the ideal of finite sets. In this case it is unknown whether there is an injective Borel representation, namely one where $E$ is equality.

Our main result is that an effective version of the completeness theorem fails at the Borel level: we build a complete consistent Borel theory without a Borel model.

We also begin to investigate Borel isomorphism between Borel structures. For instance, there are continuum many Borel presentations of $(\mathbb{R},+)$ that are not Borel isomorphic. We leave to future investigations a closer look at the "Borel dimension", the number of Borel representations that are not Borel equivalent.

We begin with some basic definitions and facts.

Definition 1.1. A set $A$ equipped with a $\sigma$-algebra $\mathcal{B}$ is said to be a standard Borel space if there is Polish topology for which $\mathcal{B}$ is the resulting class of Borel sets.

Theorem 1.2. (Kuratowski, see [Kec95]) Let $X$ be a standard Borel space and let $B \subset X$ be a Borel set. Then $B$ equipped with the canonical $\sigma$-algebra

$$
\{A \subset B: A \text { is Borel in } X\}
$$

is a standard Borel space.

Date: December 27, 2008.

Nies was partially supported by the Marsden Fund of New Zealand, grant no. 03-UOA-130. 
For us, Borel set will always mean a Borel subset of some Polish or standard Borel space equipped with the above $\sigma$-algebra of Borel subsets. The next few observations are completely routine. Details can be found in [Kec95].

Lemma 1.3. Every countable set $S$ equipped with the collection of all subsets, $\mathcal{P}(S)$, is a standard Borel space.

Consequently, we will always think of a countable set equipped with the collection of all its subsets as a standard Borel space.

Lemma 1.4. A finite product of standard Borel spaces is standard Borel.

Here we equip the finite product of standard Borel spaces, $\prod_{i \leq N} X_{i}$ with the $\sigma$-algebra generated by all cylinder sets

$$
\prod_{i \leq N} A_{i}
$$

where each $A_{i}$ is a Borel subset of $X_{i}$.

Lemma 1.5. The countable disjoint union of standard Borel spaces is again a standard Borel space.

Here we equip the countable disjoint union of standard Borel spaces, $\bigcup_{i \in \mathbb{N}}^{\cdot} X_{i}$ with the $\sigma$-algebra consisting of all sets of the form $\bigcup_{i \in \mathbb{N}}^{\cdot} A_{i}$, where each $A_{i}$ is a Borel subset of $X_{i}$.

Putting the above lemmas together, if $B$ is a standard Borel space and $S$ is a countable set, then we first obtain that $B \cup \dot{\cup} S$ is a standard Borel space in the canonical Borel structure indicated above, then that each

$$
(B \dot{\cup} S)^{N}=\prod_{i \leq N} B \dot{\cup} S
$$

is standard Borel, and then finally that

$$
(B \cup \dot{\cup} S)^{<\infty}=\bigcup_{N \in \mathbb{N}}(B \dot{\cup} S)^{N}
$$

is a standard Borel space.

Definition 1.6. A Borel signature is a Borel set $\mathcal{L}$ such that the sets

$$
\{R \in \mathcal{L}: a \text { is } \mathrm{R} \text { relation symbol of arity } n\}
$$

and

$$
\{f \in \mathcal{L}: a \text { is f function symbol of arity } n\}
$$

are all Borel. Using Polish notation one can naturally identify formulas in the resulting first order language with finite strings in

$$
\mathcal{L} \cup\left\{\neg, \vee, \wedge, \forall, \exists, v_{0}, v_{1}, \ldots\right\},
$$

where $v_{0}, v_{1}, \ldots$ are our variable symbols. In other words, the collection of well formed first order formulas, $\mathcal{L}_{\omega, \omega}$ is a subset of

$$
\left(\mathcal{L} \cup\left\{\neg, \vee, \wedge, \forall, \exists, v_{0}, v_{1}, \ldots\right\}\right)^{<\omega} .
$$

It is then easily verified that $\mathcal{L}_{\omega, \omega}$ is a Borel subset of $\left(\mathcal{L} \cup\left\{\neg, \vee, \wedge, \forall, \exists, v_{0}, v_{1}, \ldots\right\}\right)<\omega$. 
Definition 1.7. Let $\mathcal{L}$ be a Borel signature. Then a Borel first order theory in $\mathcal{L}$ is a Borel subset of $T \subset \mathcal{L}_{\omega, \omega}$, where we equip

$$
\mathcal{L}_{\omega, \omega} \subset\left(\mathcal{L} \cup\left\{\neg, \vee, \wedge, \forall, \exists, v_{0}, v_{1}, \ldots\right\}\right)^{<\infty}
$$

with the $\sigma$-algebra of Borel subsets in its canonical standard Borel structure.

Definition 1.8. We say that an equivalence relation $E$ on a standard Borel space $X$ is Borel if it is Borel as a subset of $X \times X$, in the canonical Borel structure on the product space $X \times X$. For $x \in X$ we use $[x]_{E}$ to denote the equivalence class of $x$.

Definition 1.9. Let $\mathcal{L}$ be a Borel signature. Let $\mathcal{M}$ be an $\mathcal{L}$ structure. We will say that $\mathcal{M}$ is a Borel $\mathcal{L}$ presentation if there is a standard Borel space $X$ and a Borel equivalence relation $E \subset X \times X$ such that

$$
\mathcal{M}=X / E=\left\{[x]_{E}: x \in X\right\} ;
$$

and for each $R \in \mathcal{L}$ a relation symbol of arity $n$

$$
\left\{\left(a_{0}, \ldots, a_{n-1}\right) \in X^{n}: \mathcal{M}=R\left(\left[a_{0}\right]_{E}, \ldots,\left[a_{n-1}\right]_{E}\right)\right\}
$$

is Borel as a subset of $X^{n}$; further, for each $f \in \mathcal{L}$ a function symbol of arity $n$,

$$
\left\{\left(a_{0}, \ldots, a_{n-1}, b\right) \in X^{n}: \mathcal{M}=f\left(\left[a_{0}\right]_{E}, \ldots,\left[a_{n-1}\right]_{E}\right)=[b]_{E}\right\}
$$

is Borel as a subset $X^{n+1}$. We say that a structure $\mathcal{N}$ is Borel if there is a Borel presentation $\mathcal{M}$ which is isomorphic to $\mathcal{N}$.

We will usually denote presentations as $(X, E ; \ldots)$ where $\mathcal{M}=X / E$ and the $(\ldots)$ refers to the interpretations of the various non-logical symbols of $\mathcal{L}$.

In many cases our Borel presentations will arise as structures on actual standard Borel space, rather than a quotient object of the form $X / E$. In this case we will say that the model has an injective presentation. These can also be thought of as Borel models in the above sense by taking $E$ to be the identity relation.

In [HKMN08] it is shown that some Borel structure fails to have an injective presentation. The real point of the non-completeness theorem is not just that there is a complete, consistent Borel theory with no Borel injectively presented model, but the theory in question has no Borel model even in our more generous sense.

We give some examples of Borel structures. Let $={ }^{*}$ denote the equivalence relation of eventual agreement on infinite binary sequences.

(1) The fields $(\mathbb{R},+, \times)$ and $(\mathbb{C},+, \times)$ are Borel structures. So are these fields in the extended language with names for all elements and for all continuous functions from the field to itself.

(2) All Büchi automatic structures (see [HKMN08]) are Borel structures.

(3) In particular, the Boolean algebras $\mathcal{B}=(\mathcal{P}(\mathbb{N}), \subset)$ and $\mathcal{B} /=^{*}$ are Borel structures. It is unknown whether $\mathcal{B} /=^{*}$ has an injective presentation. However, the example in [HKMN08] of a Büchi automatic structure without an injective Borel presentation is closely related to $\mathcal{B} /=^{*}$.

(4) Second order arithmetic, namely, the structure $(\mathbb{N}, \mathcal{P}(\mathbb{N}), 0,1,+, \times, \in)$, is Borel.

In fact, most structures one finds in books related to analysis such as [LZ96] are Borel. To obtain a structure of size the continuum that is not Borel one can use tools from mathematical logic: 
Proposition 1.10. [HKMN08] The linear order $\left(\omega_{1}, \leq\right)$ is not Borel.

Proof. Assume for a contradiction that $\mathcal{B}$ is a Borel presentation of $\left(\omega_{1}, \leq\right)$. Then the class of linear orderings of $\mathbb{N}$ which embed in $\mathcal{B}$ is $\underset{\sim}{\Sigma_{1}^{1}}$. This contradicts the boundedness theorem for WF [Kec95, Thm 31.2] which implies that every $\Sigma_{1}^{1}$ set of well-orderings is bounded by some ordinal $\gamma<\omega_{1}$.

A stronger result of Harrington and Shelah [HS82] states that every Borel presentable preorder can be mapped in an order preserving way into $2^{\alpha}$, the functions $\alpha \rightarrow 2$ with the lexicographical order, for some countable ordinal $\alpha$. Note that $2^{\alpha}$ is separable. Hence such a preorder has no subset of order type $\omega_{1}$.

The structure $\mathcal{B} /=^{*}$ in (3) above is an $\omega_{1}$-saturated Borel model of size the continuum for the theory of dense Boolean algebras. (This determines the Boolean algebra $\mathcal{B} /=^{*}$ up to isomorphism under $\mathrm{CH}$.) An example to the contrary is the theory of dense linear order without end points. Any of its $\omega_{1}$-saturated models of size the continuum has an $\omega_{1}$-chain, and is therefore not Borel by [HS82].

There are a few basic facts about Borel structures to which we will make repeated appeal. For instance, recalling that a set is $\sum_{1}^{1}$ if it is the Borel image of a Borel set, one has that a set is Borel if and only if both it and its complement are $\sum_{1}^{1}$. Further, a function between two standard Borel spaces is Borel measurable if and only if its graph is Borel as a subset of the product space. See [Kec95].

The following well-known theorem in Descriptive Set Theory will be essential. See Example 1.6 in [Hjo].

Theorem 1.11. There is no Borel function $F: \mathcal{P}(\mathbb{N}) \rightarrow \mathcal{P}(\mathbb{N})$ such that

$$
X={ }^{*} Y \Leftrightarrow F(X)=F(Y)
$$

for each $X, Y \subseteq \mathbb{N}$.

Acknowledgement. We thank Bakhadyr Khoussainov for his suggestion to study Borel dimension.

\section{BOREL ISOMORPHISM}

Definition 2.1. Two Borel presentations $(X, E ; \ldots),(Y, F ; \ldots)$ are said to be Borel isomorphic if there is a Borel function

$$
f: X \rightarrow Y
$$

with the following properties:

(i) for all $x_{1}, x_{2} \in X, x_{1} E x_{2}$ if and only if $f\left(x_{1}\right) F f\left(x_{2}\right)$;

(ii) for all $y \in Y$ there exists $x \in X$ with $f(x) F y$;

(iii) the function

$$
\begin{gathered}
X / E \rightarrow Y / F \\
{[x]_{E} \mapsto[f(x)]_{F}}
\end{gathered}
$$

provides an isomorphism of the two models.

A Borel structure $\mathcal{M}$ is Borel categorical if any two Borel presentation of it are Borel isomorphic. More generally, one can define the Borel dimension of a Borel structure $\mathcal{M}$ to be the number of equivalence classes modulo Borel isomorphism on the set of Borel presentations of $\mathcal{M}$. This is analogous to computable dimension in the area of recursive model theory. It was suggested by Bakhadyr Khoussainov. 
Note that $\mathcal{M}$ is Borel categorical iff it has Borel dimension 1. Presently we only know examples of Borel dimensions 1 or $2^{\aleph_{0}}$.

Examples of Borel categorical structures are:

(1) The Boolean algebra $(\mathcal{P}(\mathbb{N}), \subset)$.

(2) The linearly ordered set $(\mathbb{R}, \leq)$.

(3) The field $(\mathbb{R},+, \times)$.

For the first see [HKMN08]. For the second and third one uses the fact that the structures have isomorphic dense countable substructures. The isomorphisms between these substructures can naturally be extended to the main structures.

We show that the structure of reals under addition has the maximal Borel dimension $2^{\aleph_{0}}$.

Theorem 2.2. There are continuum many Borel presentations of $(\mathbb{R},+)$ that are not Borel isomorphic.

Proof. We exploit that any Borel isomorphism between Polish groups must be a homeomorphism (see for instance Section 1.2 of [BK96]).

For each $p>1$ recall the Banach space

$$
\ell^{p}=\left\{\vec{x} \in \mathbb{R}^{\mathbb{N}}: \sum_{n}\left|x_{n}\right|^{p}<\infty\right\},
$$

where the norm is $|\vec{x}|_{p}=\left(\sum_{n}\left|x_{n}\right|^{p}\right)^{1 / p}$. Let $G_{p}$ be the abelian group underlying $\ell_{p}$. Clearly as abstract groups these are all isomorphic, being vector spaces of dimension $2^{\aleph_{0}}$ over $\mathbb{Q}$. It suffices to show that $G_{p}$ is not Borel isomorphic to $G_{q}$ for $p \neq q$. Otherwise let $\varphi: G_{p} \cong G_{q}$ be a Borel isomorphism of Polish groups. Then, by the above remark, $\varphi$ will be a continuous, and then linear. But for $1<p<q$ there is no continuous linear bijection between $\ell^{p}$ and $\ell^{q}$. See [LZ96, top of pg. 54].

\section{Borel theories Without Borel COMPLETIONS}

The case of countable languages is special due to the following theorem of Harvey Friedman, 1979, published in [Ste85]. The idea is to use indiscernibles to obtain a large model.

Theorem 3.1. Let $T$ be a consistent first order theory in a countable language. Then $T$ has an injective Borel model of size the continuum. The model can be chosen so that its elementary diagram is Borel.

Theorem 3.1 does cannot be extended to theories over a language the size of the continuum.

Theorem 3.2. [HKMN08] There exists a consistent Borel theory which has no Borel model and no Borel completion.

Proof. Let $\mathcal{U}$ be a free ultrafilter on $\mathbb{N}$. We will consider a Borel subset of the atomic diagram of the structure $(P(\mathbb{N}), \mathcal{U})$, such that any model of it codes a free ultrafilter on $\mathbb{N}$. This contradicts the easy fact that there are no free Borel ultrafilters on $\mathbb{N}$; see for instance [Kec95, Exercise 8.50].

The signature of our theory contains a unary predicate $U$ and a constant symbol $c_{A}$ for each $A \subseteq \mathbb{N}$. The theory consist of the following sentences:

- $c_{A} \neq c_{B}$, for every $A \neq B \subseteq \mathbb{N}$;

- $U\left(c_{\mathbb{N}}\right)$ 
- $U\left(c_{A}\right) \rightarrow U\left(c_{B}\right)$, for every pair of sets such that $A \subset B \subset \mathbb{N}$;

- $U\left(c_{A}\right) \leftrightarrow \neg U\left(c_{\mathbb{N} \backslash A}\right)$, for every $A \subset \mathbb{N}$;

- $U\left(c_{A}\right) \& U\left(c_{B}\right) \rightarrow U\left(c_{A \cap B}\right)$, for every $A, B \subset \mathbb{N}$;

- $\neg U\left(c_{A}\right)$, for each finite set $A \subset \mathbb{N}$.

Clearly, this theory is Borel (even $\Pi_{1}^{0}$ ). The theory is consistent as it has the model $(P(\mathbb{N}), \mathcal{U})$ extended by constants naming each subset of $\mathbb{N}$. However, it does not have any Borel presentable model $\mathcal{X}$. Otherwise, $\left\{A \subset \mathbb{N}: c_{A}^{\mathcal{X}} \in U^{\mathcal{X}}\right\}$ is a Borel free ultrafilter on $\mathbb{N}$.

Likewise, if $T$ is a completion of our theory which is Borel, then

$$
\left\{A \subset \mathbb{N}: T \models c_{A} \in U\right\}
$$

is a Borel free ultrafilter.

We mention another example of a theorem that is proved using the axiom of choice and has no version for Borel objects: each partial order can be extended to a linear order on the same domain. The counterexample for Borel objects is due to Antonio Montalbán.

Proposition 3.3. There is a Borel relation $R \subseteq X \times X$, where $X=\{0,1\}^{\omega}$, such that $R$ is a partial order without a Borel linear extension.

Proof. Let $P$ be any Borel preorder with an $\omega_{1}$-chain, such as $\mathcal{P}(\mathbb{N})$ with almost inclusion $\subset^{*}$. We equip each equivalence class for this preorder with the lexicographical order $\leq_{l e x}$. Thus we define

$$
R x y \Leftrightarrow(P x y \& \neg P y x) \vee\left(P x y \& P y x \& x \leq_{l e x} y\right),
$$

where $x, y \subseteq \mathbb{N}$. Then $R$ is a Borel partial order that has no Borel linear extension by [HS82], as remarked after Prop. 1.10.

\section{An Anticompleteness theorem For Borel theOries}

Our main result is an "anticompleteness theorem" at the Borel level:

Theorem 4.1. There is a complete, consistent Borel theory with no Borel model.

Part of the difficulty in proving this theorem stems from the fact that we also have to rule out non-injective Borel presentations.

The proof works in stages: First we construct a Borel theory $\mathbb{T}$. Second we show that all its omega models (in a sense to be made specific later) have the same theory. Third we let $\mathbb{T}^{*}$ be the theory of those omega models, which is Borel as well. Fourth we observe that $\mathbb{T}^{*}$ has no Borel model: such a model would contain a function that contradicts Theorem 1.11.

In the following we identify $2^{\omega}$ with the collection of all subsets of $\omega$.

4.1. The theory $\mathbb{T}$. We first define a Borel theory with several sorts.

(1): $\mathcal{C}$. This includes interpretations of the constant symbols $\left\{c_{x}: x \in 2^{\omega}\right\}$. There is a relation $E$ defined on $\mathcal{C}$ with

$$
c_{x} E c_{y}
$$

if and only if $x={ }^{*} y . E$ is an equivalence relation on $\mathcal{C}$ with all equivalence classes infinite. 
$(2): \mathcal{F}$. Every element of $\mathcal{F}$ is an onto function from $\mathcal{C}$ and $B$ (where $B$ is discussed in the section on $\mathcal{T}$ below). We require that

$$
\forall f \in \mathcal{F} \forall x, y \in \mathcal{C}[x E y \Leftrightarrow f(x)=f(y)] .
$$

We require that identity in $\mathcal{F}$ is determined by its value as a function:

$$
\forall f, g \in \mathcal{F}(f \neq g \Rightarrow \exists d(f(d) \neq g(d)) .
$$

Moreover for each $n$ we introduce the axiom which states that given $d_{1}, d_{2}, \ldots, d_{n}$ in $\mathcal{C}$ which are $E$-inequivalent and given $b_{1}, \ldots, b_{n} \in B$ we have $\forall g \in \mathcal{F} \exists f \in \mathcal{F}$

$$
\left(f\left(d_{1}\right)=b_{1} \wedge f\left(d_{2}\right)=b_{2} \wedge \ldots \wedge f\left(d_{n}\right)=b_{n}\right) \wedge \forall x \not E d_{1}, \ldots, d_{n} f(x)=g(x) .
$$

(3): $\mathcal{A}$. This has constant symbols $\left(e_{n}\right)_{n \in \omega}$ and unary predicates $\left(U_{x}\right)_{x \in 2^{\omega}}$ defined over elements of $\mathcal{A}$. We will have the further sentences in our theory:

(a) Whenever $x, y, z \in 2^{\omega}$ with $z=x \cap y$ then $\forall e \in \mathcal{A}\left(\left(U_{x}(e) \wedge U_{y}(e)\right) \Leftrightarrow U_{z}(e)\right)$.

(b) Whenever $z=\omega \backslash x$ we have $\forall e \in \mathcal{A}\left(U_{x}(e) \Leftrightarrow \neg U_{z}(e)\right)$.

(c) Whenever $z \subseteq y$ we have $\forall e \in \mathcal{A}\left(U_{z}(e) \Rightarrow U_{y}(e)\right)$.

(d) If $n \notin x$ then $\neg U_{x}(n)$.

(e) If $n \in x$ then $U_{x}(n)$.

(4): $\mathcal{T}$ : This in turn consists of two types, $T$ and $B$. There is a function $L$ defined on $T$, taking values in $\mathcal{A}$. We also have a relation $Q$ defined between elements of $B$ and elements of $T$. There is a binary relation $S$ on $T$.

The intuition is as follows.

- We think of $T$ as elements of a tree.

- $S$ provides the successor relation.

- $L$ assigns levels to those elements.

- $B$ is a collection of infinite branches.

- $Q$ tells us which of the branches are above which of the nodes.

Thus we have the following axioms.

$\exists ! t \in T\left(L(t)=e_{0}\right)$.

$\forall t \in T \exists t_{0}, t_{1}\left(t_{0} S t \wedge t_{1} S t \wedge t_{0} \neq t_{1} \wedge \forall t^{\prime}\left(t^{\prime} S t \Rightarrow\left(t^{\prime}=t_{0} \vee t^{\prime}=t_{1}\right)\right)\right)$.

$\forall t, t^{\prime} \in T\left(\left(t^{\prime} S t \wedge L(t)=e_{n}\right) \Rightarrow L\left(t^{\prime}\right)=e_{n+1}\right)$.

$\forall b \in B \forall e \in \mathcal{A} \exists ! t \in T(L(t)=e \wedge Q(b, t))$.

$\forall t \in T \forall b \in B\left(Q(b, t) \Rightarrow \exists ! t^{\prime}\left(t^{\prime} S t \wedge Q(b, t)\right)\right)$.

$\forall t \in T \exists b \in B(Q(b, t))$.

$\forall b, b^{\prime} \in B\left(b \neq b^{\prime} \Rightarrow \exists t \in T\left(Q(b, t) \wedge Q\left(b^{\prime}, t\right)\right)\right)$.

We will have one last list of sentences in our theory which is designed to make the functions in $\mathcal{F}$ behave in a highly homogeneous manner. For this we introduce one further ternary relation $R$ which should be thought of as measuring the disagreement between elements of $\mathcal{F}$. $R$ will only hold when the first two coordinates are in $\mathcal{F}$ and the last in $\mathcal{A}$.

$\forall f, g \in \mathcal{F} \exists ! e \in \mathcal{A}(R(f, g, e))$.

$\forall f, g \in \mathcal{F} \forall e \in \mathcal{A}(R(f, g, e) \Rightarrow R(g, f, e))$.

$\forall f, g, h \in \mathcal{F} \forall e \in \mathcal{A}((R(f, g, e) \wedge R(g, h, e) \Rightarrow R(f, h, e))$.

For each $n$ we further introduce the sentence which says that, for all $f, g \in \mathcal{F}$, if $R\left(f, g, e_{n}\right)$ then there are exactly $n$ equivalence classes on which $f$ and $g$ disagree.

Definition 4.2. Let $\mathbb{T}$ be the above theory. $\mathcal{L}(\mathbb{T})$ denotes its language. 


\subsection{Some properties of the theory $\mathbb{T}$.}

Lemma 4.3. Each Borel model $\mathcal{M}$ of $\mathbb{T}$ is an $\omega$-model in the sense that

$$
(\mathcal{A})^{\mathcal{M}}=\left\{\left(e_{n}\right)^{\mathcal{M}}: n \in \omega\right\} .
$$

Proof. Otherwise choose $e \in(\mathcal{A})^{\mathcal{M}}$ which does not equal $\left(e_{n}\right)^{\mathcal{M}}$ for any $n \in \omega$. We obtain a Borel free ultrafilter $\mathcal{U}$ on $2^{\omega}$ by letting

$$
x \in \mathcal{U} \Leftrightarrow \mathcal{M} \models U_{x}(e),
$$

which is impossible as already mentioned after Theorem 3.2.

In the following let $T$ be a perfect binary tree (that is to say, $T$ is isomorphic to $2^{<\omega}$ under inclusion). Let $[T]$ denote the branches through $T$. We say that $S \subset[T]$ is dense if $\forall c \in T \exists b \in S[c \preceq b]$.

Lemma 4.4. (Malitz [Mal68]) Suppose the countable sets $S, S^{\prime} \subset[T]$ are dense. Then there is an automorphism $\pi$ of $T$ with $\{\pi(b): b \in S\}=S^{\prime}$.

Malitz's result is used to prove the following key lemma.

Lemma 4.5. Let $\mathcal{L}_{0}$ be a finite subset of $\mathcal{L}(\mathbb{T})$ including $\mathcal{C}, \mathcal{F}, \mathcal{A}, \mathcal{T}, E, R, L, S, Q$. Let $\mathcal{L}_{1}=\mathcal{L}_{0} \cup\left\{e_{n}: n \in \omega\right\}$. Let $\mathcal{M}_{0}, \mathcal{M}_{1}$ be countable models of $\left.\mathbb{T}\right|_{\mathcal{L}_{1}}$ which are $\omega$-models in the sense that $(\mathcal{A})^{\mathcal{M}_{i}}=\left\{\left(e_{n}\right)^{\mathcal{M}_{i}}: i \in \omega\right\}$ for $i=0,1$. Then $\mathcal{M}_{0} \cong \mathcal{M}_{1}$.

Proof. Let $\left[x_{0}\right]_{=*},\left[x_{1}\right]_{=^{*}}, \ldots,\left[x_{M}\right]_{=^{*}}$ enumerate the equivalence classes of $\left\{x: c_{x} \in\right.$ $\left.\mathcal{L}_{0}\right\}$. Choose $f \in \mathcal{F}^{\mathcal{M}_{0}}$. Choose $N$ such that for all $i \neq j \leq M$ there exists $t_{0} \neq t_{1} \in T^{\mathcal{M}_{0}}$ with

$$
\begin{gathered}
\mathcal{M}_{0} \models L\left(t_{0}\right)=e_{N}, \mathcal{M}_{0} \models L\left(t_{1}\right)=e_{N}, \\
\mathcal{M}_{0} \models Q\left(f\left(c_{x_{i}}\right), t_{0}\right), \mathcal{M}_{0} \models Q\left(f\left(c_{x_{j}}\right), t_{1}\right) .
\end{gathered}
$$

Let $T_{N}^{\mathcal{M}_{0}}, T_{N}^{\mathcal{M}_{1}}$ respectively be the sets

$$
\begin{aligned}
& \left\{t \in T^{\mathcal{M}_{0}}: \exists n \leq N \mathcal{M}_{0}=L(t)=e_{n}\right\}, \\
& \left\{t \in T^{\mathcal{M}_{1}}: \exists n \leq N \mathcal{M}_{1} \models L(t)=e_{n}\right\} .
\end{aligned}
$$

Let

$$
\sigma: T_{N}^{\mathcal{M}_{0}} \cong T_{N}^{\mathcal{M}_{1}}
$$

preserve the successor relation. Choose $b_{1}, \ldots, b_{N} \in B^{\mathcal{M}_{1}}$ such that for all $t$ with

$$
\mathcal{M}_{0} \models L(t)=e_{N},
$$

and all $i \leq M$, we have

$$
\mathcal{M}_{0} \models Q\left(f\left(c_{x_{i}}\right), t\right) \text { iff } \mathcal{M}_{1} \models Q\left(b_{i}, \sigma(t)\right) .
$$

Now let $\left(t_{i}\right)_{i \in \omega}$ enumerate nodes in $T^{\mathcal{M}_{0}}$ such that

(a) $t_{i}$ is not used in any of the branches associated to any of the $c_{x_{j}}$ 's (i.e.

$\left.\forall j \leq N \mathcal{M}_{0} \models \neg Q\left(f\left(c_{x_{j}}\right), t_{i}\right)\right)$.

(b) $t_{i}$ is an immediate successor of a branch used in some $c_{x_{j}}$ (i.e.

$\left.\exists t, j \mathcal{M}_{0} \models t_{i} S t, Q^{\mathcal{M}_{0}}\left(f\left(c_{x_{j}}\right), t\right)\right)$.

Let $\left(\widehat{t}_{i}\right)_{i \in \omega}$ be defined similarly in $T^{\mathcal{M}_{1}}$ but for the branches $b_{1}, \ldots, b_{N}$ :

(a) $\forall j \leq N \mathcal{M}_{1} \models \neg Q\left(b_{j}, \widehat{t}_{i}\right)$.

(b) $\exists t, j \mathcal{M}_{1} \models \widehat{t_{i}} S t, \mathcal{M}_{1} \models Q^{\mathcal{M}_{0}}\left(b_{j}, t\right)$.

We can do this so that

$$
\left(\mathcal{M}_{0} \models L\left(t_{i}\right)=e_{n}\right) \Rightarrow\left(\mathcal{M}_{1} \models L\left(\widehat{t_{i}}, e_{n}\right)\right),
$$


and if there exists $t$ with

$$
\mathcal{M}_{0}=Q\left(f\left(c_{x_{j}}\right), t\right), t_{i} S t
$$

then there exists $\widehat{t}$ with

$$
\mathcal{M}_{1}=Q\left(b_{j}, \widehat{t}\right), \widehat{t_{i}} S \widehat{t} .
$$

At each $i$, let $T^{i}$ be the set of $t$ in $T^{\mathcal{M}_{0}}$ which have $t_{i}$ as an ancestor. With the structure endowed by $\mathcal{M}_{0}$ this becomes a perfect binary tree - think of this intuitively as the tree of points whose last contact with one of the branches associated to one of the $x_{j}$ 's is equal to $t_{i}$. Similarly we let $\widehat{T}^{i}$ be the set of $\widehat{t} \in T^{\mathcal{M}_{1}}$ such that $\widehat{t}_{i}$ is an ancestor of $\widehat{t}$. Let

$$
\begin{aligned}
& B_{i}=\left\{b: \mathcal{M}_{0} \models Q\left(b, t_{i}\right)\right\}, \\
& \widehat{B}_{i}=\left\{b: \mathcal{M}_{1} \models Q\left(b, \widehat{t}_{i}\right)\right\} .
\end{aligned}
$$

We can then apply Malitz's lemma to find

$$
\pi: T^{i} \cong \widehat{T}^{i}
$$

with induced

$$
\pi_{i}\left[B_{i}\right]=\widehat{B}_{i}
$$

Let

$$
\pi: \mathcal{T}^{\mathcal{M}_{0}} \cong \mathcal{T}^{\mathcal{M}_{1}}
$$

be the result of patching these together and assigning

$$
\pi\left(f\left(c_{x_{j}}\right)\right)=b_{j} .
$$

Fix $\widehat{f} \in \mathcal{F}^{\mathcal{M}_{1}}$ with $\widehat{f}\left(c_{x_{j}}\right)=b_{j}$ all $j \leq M$. (The axioms listed under (2) above make this possible.)

We now extend $\pi$ to become an isomorphism of structures. For $c \in \mathcal{C}^{\mathcal{M}_{0}}$ we let

$$
\pi(c)=\left(c_{x}\right)^{\mathcal{M}_{1}}
$$

if $c=\left(c_{x}\right)^{\mathcal{M}_{0}}$ some $x$, and otherwise simply choose $\pi(c)$ so that

$$
\widehat{f}^{\mathcal{M}_{1}}(\pi(c))=\pi\left(f^{\mathcal{M}_{0}}(c)\right)
$$

this is possible since $\pi$ at the level of $B^{\mathcal{M}_{0}} \rightarrow B^{\mathcal{M}_{1}}$ is one to one and onto, and in the axioms at (1) give $\widehat{f}$ as an isomorphism between $\mathcal{C} / E$ and $B$. Since each $E$ class is infinite, we can do this so that $\pi$ provides a bijection

$$
\left([c]_{E}\right)^{\mathcal{M}_{0}} \cong\left([\pi(c)]_{E}\right)^{\mathcal{M}_{1}}
$$

The fact that $\mathcal{M}_{0}, \mathcal{M}_{1}$ are $\omega$-models in terms of $\mathcal{A}$, along with axioms at (2) ensure that $\mathcal{F}^{\mathcal{M}_{0}}$ and $\mathcal{F}^{\mathcal{M}_{1}}$ consist of exactly all the functions

$$
(\mathcal{C} / E)^{\mathcal{M}_{0}} \rightarrow B^{\mathcal{M}_{0}}
$$

and

$$
(\mathcal{C} / E)^{\mathcal{M}_{1}} \rightarrow B^{\mathcal{M}_{1}}
$$

which agree with $f$ and $\widehat{f}$ respectively on all but finitely many values. From this it is clear how to extend $\pi$ to an isomorphism between $\mathcal{F}^{\mathcal{M}_{0}}$ and $\mathcal{F}^{\mathcal{M}_{1}}$.

It only remains to define $\pi$ on $\mathcal{A}^{\mathcal{M}_{0}}$ - but here we simply send

$$
\left(e_{n}\right)^{\mathcal{M}_{0}} \mapsto\left(e_{n}\right)^{\mathcal{M}_{1}}
$$


and for the predicate symbols of the form $U_{x}$ in the common language, our axiomatization at (3) ensures we have at each $x, n$

$$
\mathcal{M}_{0} \models U_{x}\left(e_{n}\right) \Leftrightarrow \mathcal{M}_{1} \models U_{x}\left(e_{n}\right) .
$$

\subsection{The theory $\mathbb{T}^{*}$.}

Notation 4.6. Let $A_{\mathbb{T}}$ be the set of $\mathcal{M}$ as described in Lemma 4.5: That is to say, $\mathcal{M}$ is a countable $\omega$-model of $\left.\mathbb{T}\right|_{\mathcal{L}_{1}}$ for some finite subset $\mathcal{L}_{0}$ of $\mathcal{L}(\mathbb{T})$ including $\mathcal{C}, \mathcal{F}, \mathcal{A}, \mathcal{T}, E, R, L, S, Q$, and for $\mathcal{L}_{1}=\mathcal{L}_{0} \cup\left\{e_{n}: n \in \omega\right\}$. Note that $A_{\mathbb{T}}$ can be seen as a Borel set in a standard Borel space.

Definition 4.7. Let $\mathbb{T}^{*}$ be the set of $\varphi \in \mathcal{L}(\mathbb{T})$ such that there exists $\mathcal{M} \in A_{\mathbb{T}}$ with

$$
\mathcal{M} \models \varphi \text {. }
$$

Lemma 4.8. $\mathbb{T}^{*}$ is Borel.

Proof. In the light of 4.5 we have $\varphi \in \mathbb{T}^{*}$ if and only if

$$
\exists \mathcal{M} \in A_{\mathbb{T}}[\mathcal{M} \models \varphi],
$$

if and only if

$$
\forall \mathcal{M} \in A_{T}[\mathcal{L}(\varphi) \subset \mathcal{L}(\mathcal{M}) \Rightarrow \mathcal{M}=\varphi] .
$$

Thus $\mathbb{T}^{*}$ is Borel as both it and its complement are $\Sigma_{1}^{1}$.

Lemma 4.9. $\mathbb{T}^{*}$ is complete.

Proof. This is immediate by the structure of the definition of $\mathbb{T}^{*}$.

Lemma 4.10. $\mathbb{T}^{*}$ is consistent.

Proof. From 4.5 and the definition of $\mathbb{T}^{*}$, we have that for any finite $\mathcal{L}_{0} \subset \mathcal{L}\left(\mathbb{T}^{*}\right)$ there is a countable model of $\left.\mathbb{T}^{*}\right|_{\mathcal{L}_{0}}$.

Theorem 4.11. $\mathbb{T}^{*}$ has no Borel model.

Proof. First we show that there is no injective Borel model $\mathcal{M}$ of $\mathbb{T}^{*}$. Assume otherwise. By lemma 4.3 and $\mathbb{T}^{*} \supset \mathbb{T}$,

$$
(\mathcal{A})^{\mathcal{M}}=\left\{\left(e_{n}\right)^{\mathcal{M}}: n \in \omega\right\} .
$$

Let $\left(t_{n}\right)_{n \in \omega}$ enumerate elements of $(T)^{\mathcal{M}}$. For each $b \in B^{\mathcal{M}}$, we let

$$
\rho(b)=\left\{n: \mathcal{M}=Q\left(b, t_{n}\right)\right\} .
$$

Fix $f \in(\mathcal{F})^{\mathcal{M}}$. Define $\theta: 2^{\omega} \rightarrow 2^{\omega}$ by

$$
\theta(x)=\rho\left(\left(f\left(c_{x}\right)\right)^{\mathcal{M}}\right) .
$$

Then for all $x, y \in 2^{\omega}$,

$$
x={ }^{*} y \Leftrightarrow \mathcal{M} \models c_{x} E c_{y},
$$

by the axioms at (1),

$$
\Leftrightarrow \mathcal{M} \models f\left(c_{x}\right)=f\left(c_{y}\right),
$$

by the axioms at (2),

$$
\Leftrightarrow \rho\left(\left(f\left(c_{x}\right)\right)^{\mathcal{M}}\right)=\rho\left(\left(f\left(c_{y}\right)\right)^{\mathcal{M}}\right),
$$


by the axioms at (4) describing $B$ as a collection of branches through $T$. Thus we obtain a Borel function $\theta$ with

$$
x={ }^{*} y \Leftrightarrow \theta(x)=\theta(y),
$$

contradicting Theorem 1.11.

As already mentioned above, even if we allow Borel models where equality in $\mathcal{M}$, $(=)^{\mathcal{M}}$, does not actually correspond to true $=$ in the outside world, there still is no Borel model of $\mathbb{T}^{*}$. The only adjustment to the above argument is to let $\left(t_{n}\right)_{n \in \omega}$ be a complete sequence of representatives for elements of $(T)^{\mathcal{M}}$.

\section{REFERENCES}

[BK96] Becker and Kechris. The descriptive set theory of Polish group actions. Cambridge University Press, 1996.

[Hjo] Greg Hjorth. Borel equivalence relations. To appear in the Handbook on Set Theory edited by Matt Foreman and Aki Kanamori.

[HKMN08] G. Hjorth, B. Khoussainov, A. Montalban, and A. Nies. From automatic structures to borel structures. In Proceedings of the 19th IEEE Symposium on Logic in Computer Science, Lecture Notes in Computer Science, pages 110-119. IEEE Computer Society, 2008.

[HS82] Leo Harrington and Saharon Shelah. Counting equivalence classes for co- $\kappa$-Souslin equivalence relations. In Logic Colloquium '80 (Prague, 1980), volume 108 of Stud. Logic Foundations Math., pages 147-152. North-Holland, Amsterdam, 1982.

[Kec95] Alexander S. Kechris. Classical descriptive set theory, volume 156 of Graduate Texts in Mathematics. Springer-Verlag, New York, 1995.

[LZ96] B. Lindenstrauss and K. Zafiri. Classical Banach Spaces I. Springer, 1996.

[Mal68] J Malitz. The hanf number for complete $l_{\omega_{1}, \omega}$ sentences. In The syntax and semantics of infinitary languages, Lecture Notes in Mathematics, pages 166-181. Springer, 1968.

[Ste85] C. I. Steinhorn. Borel structures and measure and category logics. In Model-theoretic logics, Perspect. Math. Logic, pages 579-596. Springer, New York, 1985.

Department of Mathematics and Statistics, The University of Melbourne, Melbourne, Australia

E-mail address: greg.hjorth@gmail.com

Department of Computer Science, Auckland University, Auckland, New Zealand

E-mail address: andre@cs.auckland.ac.nz 